\title{
On dewetting dynamics of solid films of hydrogen isotopes and its influence on tritium $\beta$ spectroscopy
}

\author{
L. Fleischmann ${ }^{1}$, J. Bonn ${ }^{1}$, B. Bornschein ${ }^{1}$, P. Leiderer ${ }^{2}$, E.W. Otten ${ }^{1}$, M. Przyrembel ${ }^{1}$, and Ch. Weinheimer ${ }^{1, a}$ \\ 1 Institute of Physics, Johannes Gutenberg University, 55099 Mainz, Germany \\ 2 Faculty of Physics, Konstanz University, 78457 Konstanz, Germany
}

Received 30 December 1999

\begin{abstract}
The dewetting dynamics of solid films of hydrogen isotopes, quench-condensed on a graphite substrate, was measured at various temperatures below desorption by observing the stray light from the film. A schematic model describing the dewetting process by surface diffusion is presented, which agrees qualitatively with our data. The activation energies of different hydrogen isotopes for surface diffusion were determined. The time constant for dewetting of a quench-condensed $\mathrm{T}_{2}$ film at the working temperature of $1.86 \mathrm{~K}$ of the mainz neutrino mass experiment was extrapolated.
\end{abstract}

PACS. $67.80 \mathrm{Mg}$ Defects, impurities and diffusion $-67.70 .+\mathrm{n}$ Films (including physical adsorption) 68.45.Gd Wetting - 23.40.-s $\beta$-decay; double $\beta$ decay; electron and muon capture

\section{Introduction}

Molecular hydrogen exhibits the effect of triple point wetting (for a review on wetting phenomena see, e.g. [1]). This implies that although liquid films above the triple point temperature $T_{\mathrm{t}}$ can grow to arbitrary thickness, solid $\mathrm{H}_{2}$ well below $T_{\mathrm{t}}$ in thermal equilibrium forms on a given substrate only homogeneous films of at most a few monolayers $[2,3]$. Any additional material dewets from this residual film and clusters to microscopic crystals. We have investigated conditions under which this effect could be prevented, since it hampers the use of solid hydrogen films as well-defined matrix or substrate material.

In this respect our team is particularly involved in precision measurements of the endpoint region of the $\beta$ spectrum from a source of frozen tritium molecules $T_{2}$ in search for a possible rest mass $m_{\nu}$ of the neutrino, emitted simultaneously [4]. The analysis of the $\beta$ spectrum requires amongst others a precise knowledge of the energy loss which the particles suffer in traversing the $\mathrm{T}_{2}$ source. Clearly this depends on its geometry. In a flat film of say 50 monolayers electrons, emitted up to a maximum angle of $\vartheta_{\max }=78^{\circ}$ (see Eq. (2)), suffer single inelastic scattering with $12.8 \%$ probability, whereas multiple inelastic scattering occurs in only $1.4 \%$ of the cases. Within the much thicker clusters of a dewetted source of same average thickness, however, these probabilities are increased, leading to a higher average energy loss per emitted electron as well as to a shift of the energy loss spectrum towards higher energy losses. In fitting the $\beta$ spectrum underestimation of energy loss correlates with $m_{\nu}^{2}$ and leads to unphysical,

a e-mail: christian.weinheimer@uni-mainz.de negative values of $m_{\nu}^{2}$. In our first measurement of $m_{\nu}^{2}$ in $\mathrm{T}_{2}$ decay [5] we came across this complication but did not yet identify dewetting as the origin of enhanced energy loss.

In Section 2 we present energy loss measurements of $18 \mathrm{keV}$ electrons in $\mathrm{D}_{2}$ films at temperatures of $2.8 \mathrm{~K}$ and $4.2 \mathrm{~K}$. At the higher temperature they clearly evidence enhanced energy loss pointing to a dewetted state [6].

Our attempts to stabilise homogeneous films of frozen hydrogen molecules were twofold. The first one was directed towards a static stabilisation, i.e. we searched for a substrate with a suitable relative adsorption strength $1.8 \leq R \leq 3$ at which at any thickness the flat wetting phase should be energetically favoured over the dewetted, clustered one, according to theory [7]. Here $R$ is the ratio of the van der Waals potential between substrate and condensate to the one within the condensate itself. This expectation could not be confirmed, neither for pure substrates nor for sandwiched ones where $R$ was modified by interfacing a few monolayers of a third substance in between the substrate and the condensate. In all cases investigated, the hydrogen film dewetted. We have reported on these findings in a foregoing paper [8]. In a second attempt we have investigated conditions under which the quench-condensed films could be stabilised at least dynamically; i.e. we measured the temperature dependence of dewetting time constants. Thus we found temperature limits below which dewetting is sufficiently slow. We report on these experiments in Section 3. They differ from earlier dynamical studies of hydrogen dewetting [9-11] in the method of signal detection and also include measurements on $\mathrm{T}_{2}$ films for the first time. 
In Section 4 we present a schematic model which treats the process of dewetting and cluster growth in terms of surface diffusion on the residual film between the boundaries of still quench-condensed, disordered ${ }^{1}$ adsorbate and those of the energetically favoured crystalline clusters. That surface, not volume diffusion drives the dewetting has been proven unambiguously by covering the hydrogen condensates with some monolayers of other, slightly tighter bound van der Waals systems like $\mathrm{Ne}$, Ar, or $\mathrm{CH}_{4}$ which definitely prevented the hydrogen film from dewetting $[14,8]$. The analysis of experimental data in Section 5 yields the decisive activation energies of the process for the different isotopes and proves, moreover, that the schematic model is in satisfactory qualitative agreement with the various features observed.

\section{Observation of enhanced energy loss of fast electrons in dewetting $D_{2}$ films}

After having learned that quench-condensed films of hydrogen isotopes may dewet from the substrate to form much thicker clusters [9] we have checked the influence of this phenomenon on the energy loss of fast traversing electrons with our integrating $\beta$-spectrometer [15]. This instrument collimates charged particles emitted isotropically from a source by magnetic a diabatic collimation which allows to analyse very sharply their energy spectrum over the full $2 \pi$ solid angle of forward emission by a subsequent electrostatic filter at a potential $U$ (MAC-Efilter). Particles of charge $q$ with energy $E>q U$ pass the potential barrier and are refocused magnetically on a detector. The relative filter width $\Delta E / E$, within which the transmission rises from 0 to 1 equals the ratio of the magnetic field $B_{\mathrm{a}}$ in the analysing plane at potential $U$ to the maximal field $B_{\max }$ which is achieved in between source and analysing plane (see Fig. 1). For the measurements presented here we have chosen a relative resolution of

$$
\Delta E / E=B_{\mathrm{a}} / B_{\max }=1 / 3000 .
$$

The field $B_{\mathrm{s}}$ at the source has been chosen somewhat weaker than $B_{\max }$, such that particles, emitted from the source under polar angles

$$
\theta>\theta_{\max }=\arcsin \left(\sqrt{B_{\mathrm{s}} / B_{\max }}\right)=78^{\circ}
$$

are reflected back to the source by the magnetic mirror effect. With this truncation of the accepted solid angle the average path length through a transverse target of thickness $d$ is calculated to be $\bar{d}=2 d$.

\footnotetext{
1 The degree of disorder will depend on the condensation conditions. It is known from rare gas systems that with experimentally attainable quenching rates not really amorphous, but rather nanocrystalline films are formed [12], whose the structural details, like the porosity, are influenced by the preparation procedure [13]. In the stage of clustering discussed below these details do not appear to be relevant, however.
}

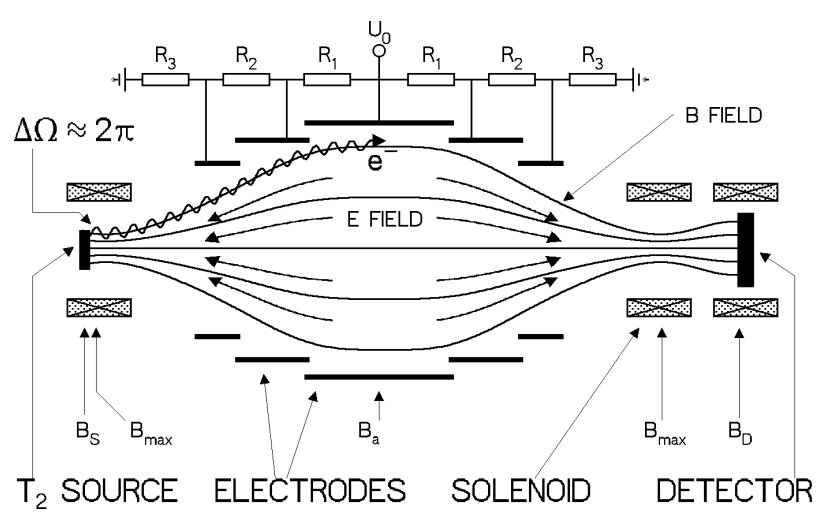

Fig. 1. Sketch of the mainz solenoid retarding spectrometer.

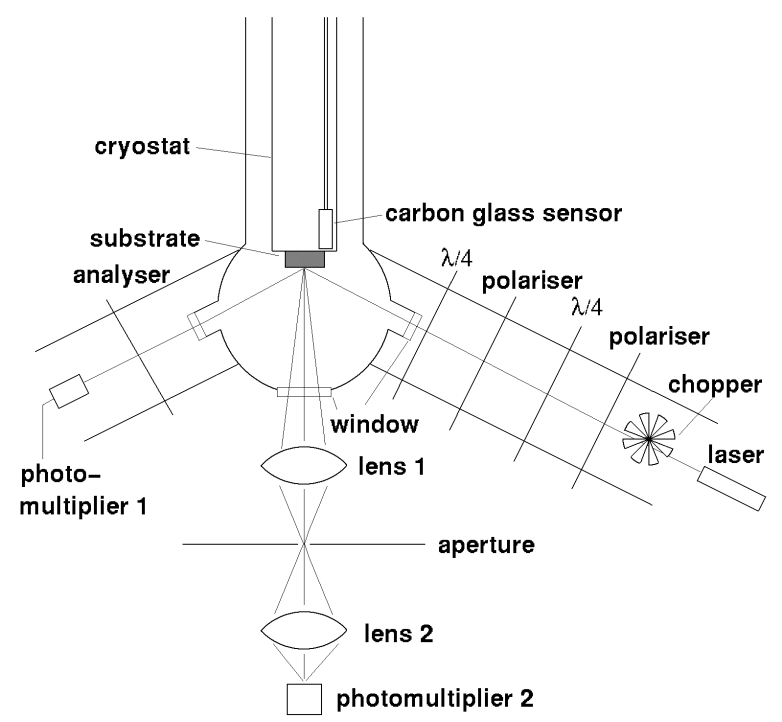

Fig. 2. Experimental set-up for investigating the quenchcondensed films. The ellipsometer in a typical, Polariser Substrate Compensator Analyser configuration measures the film thickness. The first polarizer $\lambda / 4$ combination in the laser beam is used for generating circularly polarised light. Photomultiplier 2 detects the stray light under $60^{\circ}$ and $120^{\circ}$. The chopper in the laser beam allows a very sensitive detection by a lock-in amplifier.

As electron source with an energy close to that of the endpoint of $\mathrm{T}_{2} \beta$-decay at $18.6 \mathrm{keV}$ we have chosen the $17.8 \mathrm{keV}$ K32 conversion line of the krypton isomer ${ }^{83 m} \mathrm{Kr}$, with a half life of $1.83 \mathrm{~h}$. The source is quasi monochromatic, i.e. the main peak at full energy has a Lorentzian width of $2.8 \mathrm{eV}$ FWHM and is followed by shake up and shake off satellites at lower energies, the closest being the $\mathrm{N}$ shake up about $20 \mathrm{eV}$ apart (for details see e.g. Ref. [16]). A submonolayer of ${ }^{83 m} \mathrm{Kr}$ was condensed on a cold graphite substrate (HOPG) and covered with quenchcondensed $\mathrm{D}_{2}$ films about $80 \AA$ thick. The thickness was controlled by standard laser ellipsometry (see Fig. 2). Note that ellipsometry does not resolve the microscopic roughness of a dewetted $\mathrm{D}_{2}$ film since the transmitted and reflected beams measure the average optical thickness of the layer. 


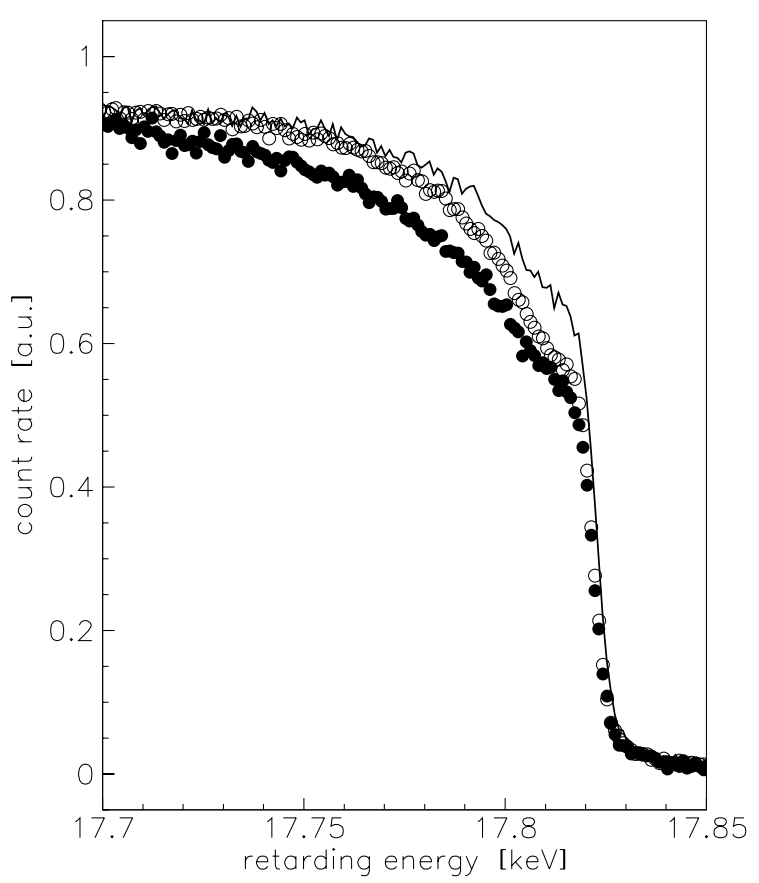

Fig. 3. Intensity (normalized at $17.60 \mathrm{keV}$ ) of transmitted K32 conversion electrons from a ${ }^{83 m} \mathrm{Kr}$ source as function of the filter potential $q U$ for three cases: (i) a naked ${ }^{83 m} \mathrm{Kr}$ source (line), (ii) an $82 \AA \mathrm{D}_{2}$ film quench-condensed and then measured at a temperature $T=2.8 \mathrm{~K}$ (circles), (iii) an $88 \AA \mathrm{D}_{2}$ film quench-condensed and measured at $T=4.2 \mathrm{~K}$ (dots).

Figure 3 shows the intensity of transmitted electrons as function of the filter potential for three cases: (i) a naked ${ }^{83 m} \mathrm{Kr}$ source (line), (ii) an $82 \AA \mathrm{D}_{2}$ film quench-condensed and then measured at a temperature $T=2.8 \mathrm{~K}$ (circles), (iii) an $88 \AA \mathrm{D}_{2}$ film quench-condensed and measured at $T=4.2 \mathrm{~K}$ (dots). The common steep rise at $17.825 \mathrm{keV}$ occurs when the filter opens to the elastic $\mathrm{K}$ conversion peak. After reaching a plateau the intensity rises further when the filter opens to the first electronically excited states of $\mathrm{D}_{2}$ at about $15 \mathrm{eV}$ [17] and to the closest shake up satellites at $20 \mathrm{eV}$. Lowering the filter potential further, the intensity rises smoothly until the entire energy loss spectrum is integrated up and the filter is open for all electrons. This situation is virtually achieved - at least with respect to losses in the $\mathrm{D}_{2}$ film - at the left end of the curves at an energy loss of $\varepsilon \approx 230 \mathrm{eV}$. Therefore, at that point the three curves are normalised to equal intensity.

Comparing the integral spectrum of the warmer to that of the colder film, one clearly recognises that in the warmer case intensity is shifted from lower to higher energy losses. Only a small fraction of this effect can be ascribed to the somewhat larger average thickness $(6 \AA$ more) of the warmer film. The majority must be due to a structural change of the film in the sense of a clustering of the material. The analysis of the difference spectrum may be summarised as follows: An average of about $5 \%$ of the electrons has suffered in the warmer film an additional energy loss of about $\varepsilon=86 \mathrm{eV}$ in the average.
The enhanced energy loss in a dewetted $\mathrm{D}_{2}$ film, which has been observed here in a transmission experiment, is also in accordance with the one observed in our first $\mathrm{T}_{2}$ decay experiment [5]. There an additional energy loss of $\varepsilon \approx 75 \mathrm{eV}$ with a probability of $4 \%$ per decay has been inferred from the observed anomaly in the $\beta$ spectrum. Note that an excess of energy loss in this region of the spectrum correlates to $m_{\nu}^{2}$ in the fit to first order by its mean value only. This value is about the same in both cases, although one would expect some difference since the electron emitting $\mathrm{Kr}$ layer is placed below the clusters in the present case, whereas the decay electrons from tritium are emitted from the clusters itself.

According to these results the spectral anomaly observed in our first experiment on tritium decay [5] can now be fully explained by the assumption that the $\beta$-particles emerged from a dewetted $\mathrm{T}_{2}$ film. What is called here a $\mathrm{T}_{2}$ film was in reality a mixture of tritium and hydrogen with a dewetting dynamics close to that of $\mathrm{D}_{2}$ (see Sect. 3 ). It had been quench-condensed at $4.2 \mathrm{~K}$ like the $\mathrm{D}_{2}$ film in the transmission experiment and, therefore, certainly dewetted according to our present knowledge. In our recent measurements since 1997 where a new cryostat guarantees a temperature below $2 \mathrm{~K}$ the films are stable and the problem is gone [4].

\section{Dewetting dynamics observed by light scattering}

The energy loss measurements described in the preceding section clearly distinguished between homogeneous and dewetted films. However, they did not allow to observe the dewetting process in real time. For this purpose light scattering experiments are well suited on which we report in the following section.

The device for observing light scattering from quenchcondensed hydrogen films could be easily integrated into the ellipsometry set-up (see Fig. 2). Stray light from the laser spot on the film was imaged in normal direction onto a photomultiplier. The scattering angles with respect to the incident and the reflected laser beam were $120^{\circ}$ and $60^{\circ}$, respectively. For the measurements described here we used a polished aluminium substrate, since it produces less background light than the graphite substrate which is preferred in the $\beta$ spectroscopy experiments for its lower electron backscattering, on the other hand. The films were condensed at a rate of $10 \mathrm{~nm} / \mathrm{min}$ typically, with the substrate temperature being kept at values between $1.6 \mathrm{~K}$ and $2.5 \mathrm{~K}$. The temperature of the condensing gas could not be measured, but since the feeding capillary was cooled only at its end by radiation cooling, we assume that is was at least $20 \mathrm{~K}$ or even higher. During film preparation a shielding cap covered the substrate to contain the gas. It had an inlet and outlet window for the laser beam which allowed ellipsometric control of the film growth during condensation.

In the next step the substrate temperature was increased to a value where the relaxation process of the film 
was to be studied. The intensity of the scattered light, which gives a measure of the growing film roughness upon annealing, was then registered as a function of time. Once the relaxation process had come to an end, the substrate temperature was increased further so that the film desorbed, and the scattering background of the substrate could be determined. A comparison with the scattering signal from the fresh film allowed then to draw conclusions about the existence of scattering centres in the quenchcondensed film from the beginning, like porosity and larger defects. Usually the background level was the same in both cases indicating that porosity and roughness of the fresh, quench-condensed films have length scales very far below the wavelength of $632.8 \mathrm{~nm}$ of the HeNe laser. By growing thick $\mathrm{D}_{2}$ films, which allowed to measure thickness $d$ and refraction index $n$ of the film separately, we learned, on the other hand, that the quench-condensed films exhibit a porosity of $11 \%$ compared to compact, solid $\mathrm{D}_{2}$. This was seen from a reduction of $n$ to a value of $1.1415 \pm 0.0020$, as compared to the value of 1.159 obtained from the literature value for the molecular volume of $\mathrm{D}_{2}$ of $V_{\mathrm{mol}}\left(\mathrm{D}_{2}\right)=19.95 \mathrm{~cm}^{3}$ [18] applying Clausius-Mosotti's law. It is known that the porosity of quench-condensed films depends on the conditions of preparation. Slower condensation at higher gas temperature leads to more compact films. Porosity may influence stability and dewetting dynamics of the films. Since we grew our films differently from preceding experiments on hydrogen dewetting $[10,11]$, one may expect somewhat different results as well.

The relaxation of the films was observed at various fixed temperatures between $2.5 \mathrm{~K}$ and $3.3 \mathrm{~K}$ for $\mathrm{H}_{2}, 3.0 \mathrm{~K}$ and $3.8 \mathrm{~K}$ for $\mathrm{HD}, 3.8 \mathrm{~K}$ and $4.4 \mathrm{~K}$ for $\mathrm{D}_{2}, 4.2 \mathrm{~K}$ and $4.8 \mathrm{~K}$ for " $\mathrm{T}_{2}$ ". The isotopic composition of the " $\mathrm{T}_{2}$ " gas, which was quench-condensed on the substrate, was determined by a quadrupole mass filter to be: $21 \% \mathrm{H}_{2}, 38 \%$ $\mathrm{HT}$, and $41 \% \mathrm{~T}_{2}{ }^{2}$. Temperatures were determined by a carbon glass thermometer. The exponential characteristics of its resistance leads to a steep increase of sensitivity with lower temperature. It was calibrated by measuring hydrogen desorption rates whose constants are well-known. Errors in temperature were below $0.1 \mathrm{~K}$ at the lower end and reached $0.2 \mathrm{~K}$ at the upper end of the temperature interval where the film relaxed quite fast within minutes already. It is also for that reason that we preferred an aluminium substrate for these dynamic measurements, since the poor thermal conductivity of the HOPG graphite leads to considerable temperature gradients between its faces.

As an example for the acceleration of dewetting with temperature, Figure 4 shows the time dependence of the stray light from $\mathrm{D}_{2}$ films observed at $3.8 \mathrm{~K}, 4.0 \mathrm{~K}$ and $4.4 \mathrm{~K}$, respectively. The time scale $\Delta t$ within which dewetting develops changes from about 1 hour to a couple of minutes. It is obviously governed by an Arrhenius law characterised by an activation energy $\Delta E$. From this $\Delta E$ we may predict in a straightforward way that relaxation

\footnotetext{
2 The composition of the quench-condensed film should be very similar to that of the gas, since the measured optical refraction index agrees well with the one expected for the gas composition.
}

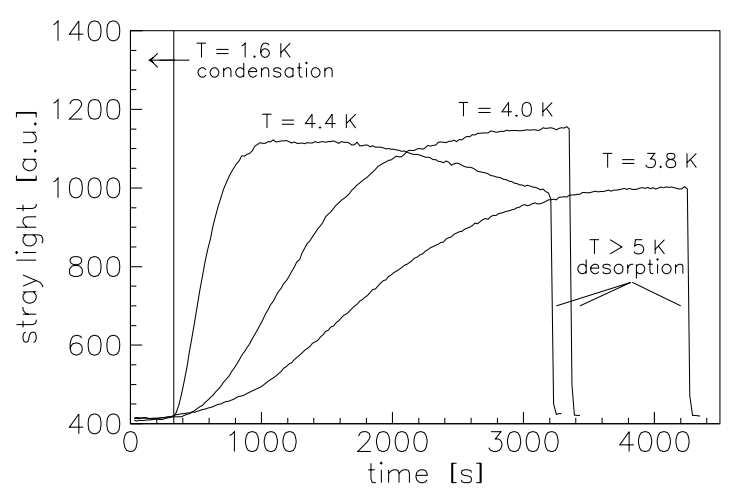

Fig. 4. Stray light intensity versus time measured for $\mathrm{D}_{2}$ films of similar thickness but at different dewetting temperatures (film 1: $184 \AA, T_{\text {dewet }}=4.4 \mathrm{~K}$, film 2: $160 \AA, T_{\text {dewet }}=4.0 \mathrm{~K}$, film 3: $\left.109 \AA, T_{\text {dewet }}=3.8 \mathrm{~K}\right)$. The temperature was raised from $1.6 \mathrm{~K}$ to the dewetting temperature at $330 \mathrm{~s}$, and raised again to a temperature $T>5 \mathrm{~K}$ at times marked between $3000 \mathrm{~s}$ and $4500 \mathrm{~s}$.

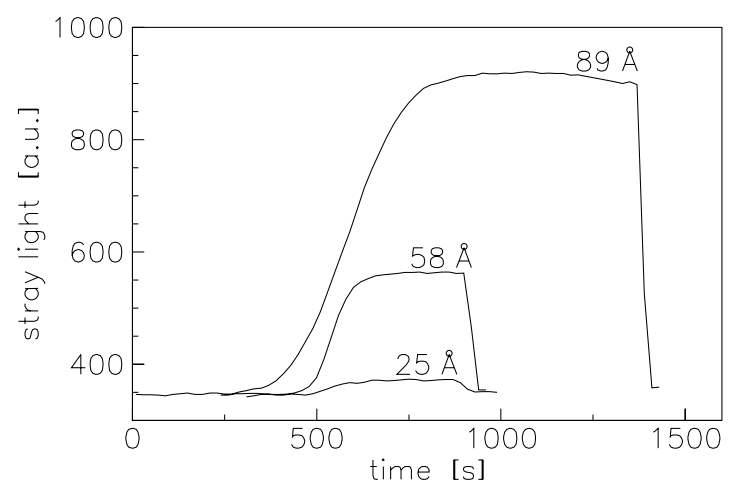

Fig. 5. Stray light intensity versus time for $D_{2}$ films of various thickness at the same dewetting temperature of $4.2 \mathrm{~K}$.

times will become virtually infinitely long below $2 \mathrm{~K}$. Figure 5 shows the development of the stray light signal for $\mathrm{D}_{2}$ films of different thickness, the time scale of dewetting and in particular the stray light growth with film thickness.

Further analysis will be given in terms of a surface diffusion model introduced in the following section.

\section{Surface diffusion model of dewetting}

At the present stage of knowledge it would be premature to aim at an exact theory of the observed dewetting phenomena of hydrogen films. Let us try, therefore, to describe the clustering by a schematic model. It assumes surface diffusion between energetically disfavoured regions of quench-condensed, disordered, porous hydrogen and crystallised clusters. The latter ones are assumed to be energetically favoured to the extent that back diffusion across this energy barrier is suppressed. So they grow unilaterally on expense of the disordered material. We further assume that initial crystallites have grown in relatively short time to the size of film thickness and are sitting within a "depletion crater" of originally disordered material which they 


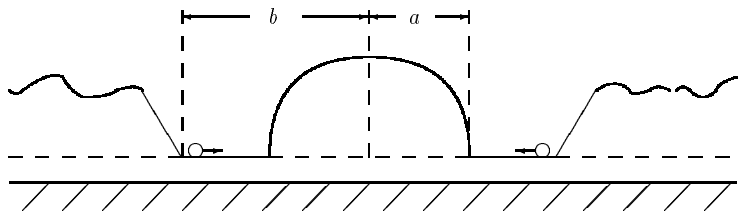

Fig. 6. Schematic model of film clustering by surface diffusion of molecules from a disordered film to crystallites of radius $a$ on top of a residual film.

have eaten up already. Further transport is then by surface diffusion on the bottom of this crater which is a residual thin hydrogen layer, tightly bound to the substrate (see Fig. 6). The diffusion constant is derived from a classical hopping model on a lattice with lattice constant $g$ (which for simplicity we assume to be quadratic) and identical hopping rates $\nu_{x}=\nu_{y}=\nu$ in both directions given by Frenkel's law

$$
\nu=\nu_{0} \mathrm{e}^{-\Delta E / k_{\mathrm{B}} T}
$$

with $\nu_{0}$ being the vibrational frequency of a molecule on a lattice site of the surface and $\Delta E$ being the activation energy of hopping to a neighbouring site. If we assume the latter to form a simple periodic potential

$$
E_{p}(x, y)=\Delta E \sin ^{2}\left(\frac{\pi x}{g}\right) \sin ^{2}\left(\frac{\pi y}{g}\right),
$$

we obtain in harmonic approximation

$$
\nu_{0}=\frac{1}{g} \sqrt{\frac{\Delta E}{2 m}} .
$$

After a diffusion time $t$ the mean squared displacements in both directions are then

$$
\overline{X^{2}}=\overline{Y^{2}}=g^{2} \nu t
$$

from which we derive the two-dimensional surface diffusion constant

$$
D_{\mathrm{s}}=\frac{1}{2}\left(\overline{X^{2}}+\overline{Y^{2}}\right) / t=g^{2} \nu=g^{2} \nu_{0} \mathrm{e}^{-\Delta E / k_{\mathrm{B}} T} .
$$

To give an example, we may set $\Delta E$ equal to the activation energy of $47 k_{\mathrm{B}} \mathrm{K}$, which Classen et al. found in a different experiment for dewetting of $\mathrm{D}_{2}$ films [11]. With $m_{\mathrm{D}_{2}}=6.6 \times 10^{-24} \mathrm{~kg}$ and $g=3.4 \AA$ [19] one finds for the vibrational frequency

$$
\nu_{0 \mathrm{D}_{2}}=6.5 \times 10^{11} / \mathrm{s},
$$

for the hopping rate at $4 \mathrm{~K}$ according equation (3)

$$
\nu_{\mathrm{D}_{2}}(4 \mathrm{~K})=5.1 \times 10^{6} / \mathrm{s},
$$

and for the diffusion constant

$$
D_{\mathrm{s}}=5.9 \times 10^{-13} \mathrm{~m}^{2} / \mathrm{s} .
$$

We calculate the diffusion current $I_{\mathrm{S}}$ for a simplified geometry between circular boundaries of a crater with radius $b$ and a hemispherical cluster ${ }^{3}$. At given radius $a<r=b$ the diffusion equation then reads

$$
j_{\mathrm{s}}=\frac{I_{\mathrm{s}}}{2 \pi r}=-D_{\mathrm{s}} \frac{\mathrm{d} n_{\mathrm{s}}}{\mathrm{d} r}
$$

with $j_{\mathrm{s}}, n_{\mathrm{s}}$ being surface current and number density, respectively, of the diffusing particles. With the afore mentioned boundary condition $n_{\mathrm{s}}(a)=0$ (no back diffusion) the solution of (11) is

$$
I_{\mathrm{s}}=2 \pi D n_{\mathrm{s}}(b) / \ln (b / a) .
$$

The ratio $b / a$ is fixed by equalling the volume of the hemisphere to that of the crater which we approximate by $\pi b^{2} d$ giving

$$
\frac{b}{a}=\sqrt{\frac{2}{3 d}\left(\frac{3 N}{2 \pi \rho}\right)^{1 / 3}}
$$

with $N$ being the number of molecules in the hemisphere and $\rho=3.0 \times 10^{28} / \mathrm{m}^{3}$ being the bulk number density of solid $\mathrm{D}_{2}$. Equation (13) has to obey the condition $b / a>1$ and hence requires a minimum $N$ at given $d$. Substituting (13) into (12) yields the differential equation of the growth

$$
I_{\mathrm{s}}=\dot{N}=\frac{12 \pi D_{\mathrm{s}} n_{\mathrm{s}}(b)}{\ln \left(4 N / 9 \pi \rho d^{3}\right)},
$$

whose solution reads

$$
\begin{aligned}
\Delta t= & t_{1}-t_{0} \\
= & \frac{1}{12 \pi D_{\mathrm{s}} n_{\mathrm{s}}(b)} \int_{N_{0}}^{N_{1}} \ln \left(\frac{4}{9 \pi \rho d^{3}} N\right) \mathrm{d} N \\
= & \frac{\mathrm{e}^{\Delta E / k_{\mathrm{B}} T}}{12 \pi g^{2} \nu_{0} n_{\mathrm{s}}(b)} \\
& \times\left(N_{1}\left(\ln \left(\frac{4 N_{1}}{9 \pi \rho d^{3}}\right)-1\right)-N_{0}\left(\ln \left(\frac{4 N_{0}}{9 \pi \rho d^{3}}\right)-1\right)\right) .
\end{aligned}
$$

In (15) we have used for $D_{\mathrm{s}}$ the explicit formula (7). We may collect all temperature-independent terms in a single effective time constant $\tau_{0 \text {,eff }}$ and get for the dewetting time $\Delta t$ an effective Frenkel law

$$
\Delta t=\tau_{0, \mathrm{eff}} \mathrm{e}^{\Delta E / k T} .
$$

Classen et al. [11,20] and Conradt et al. [21,22] have started the analysis of their dewetting experiments directly from an ansatz according (16). For a $\mathrm{H}_{2}$-film of $\mathcal{O}(100) \AA$ thickness Conradt et al. [21] have found a value $\tau_{0, \text { eff }}=3.4 \times 10^{-3} \mathrm{~s}$ which is of course much larger than

${ }^{3}$ This simplified picture probably does not describe the exact shape and the exact contact angle of the cluster on the residual $\mathrm{D}_{2}$ film (see Fig. 6), which unfortunately are not known. Nevertheless the essential features of our rough model do not depend on the exact knowledge of these parameters. 
the vibrational period $1 / \nu_{0}($ see $(5,7))$, since it relates to the total dewetting process, not to the single hopping.

Our model yields for $\tau_{0 \text {,eff }}$ the explicit formula (15). It is still proportional to the hopping time constant $1 / \nu_{\mathrm{o}}$, as expected, but depends in addition on a few more parameters:

- the surface density $n_{\mathrm{s}}(b)$ of diffusing particles at the crater boundary. Here we use the upper bound of the porous surface $n_{\mathrm{s}}(b)=10^{19} / \mathrm{m}^{2}$ as an estimate.

- a linear dependence on the final (average) number $N_{1}$ contained in a cluster. The sharp increase of stray light with film thickness $d$, displayed in Figure 5 , proves that the final cluster size and hence $N_{1}$ clearly increases with $d$. The corresponding increase of $\Delta t$ or $\tau_{0 \text {,eff }}$, respectively, is also seen.

- a logarithmic dependence on

$$
\frac{N_{1}}{d^{3} \rho}=\frac{1}{d^{2}(\mathrm{~d} Z / \mathrm{d} A)}
$$

where $\mathrm{d} Z / \mathrm{d} A$ is the number of primary crystallisation nuclei per unit area. It again reflects the weak logarithmic dependence of the rate of growth (12) on the ratio of sizes $b / a$.

\section{Analysis and discussion of results}

\subsection{Some consequences of the diffusion model}

We will start this section by discussing some properties and consequences of our schematic diffusion model. First we have to point to its obvious shortcoming in the initial phase of clustering when the crater radius $b$ is still close to the cluster radius $a$. There the diffusion current $I_{\mathrm{S}}$ diverges logarithmically as $1 / \ln (b / a)$. That is a consequence of our extreme boundary conditions which for simplicity neglect any diffusional resistivity within the source at $r>b$ and within the drain at $r<a$. Anyway, this initial phase is relatively short compared to the total time of growth and does not result in a detectable roughening signal yet.

On the other hand, the logarithmic dependence of (12) on the size of the boundaries is inherent to surface diffusion and, therefore, will characterise also the exact solution of the problem. Consequently, the rate of growth of a cluster decreases only moderately with its size, e.g. by a factor 2.3 between $b / a=2$ and $b / a=5$. Starting with a film thickness of $d=130 \AA$, the latter ratio corresponds already to a cluster size of $a=0.5 \mu \mathrm{m}$ which is about the final value observed $[20,21]$. We may, therefore, simplify our qualitative analysis by assuming an average, constant rate (12) for the decisive phase of growth. Inserting into (12) the diffusion constant (10) from our example of a $\mathrm{D}_{2}$ film at $4 \mathrm{~K}$ and the maximum surface density $n_{\mathrm{s}}(b)=10^{19} / \mathrm{m}^{2}$, we calculate for an intermediate ratio $b / a=3.5$ a rate of growth of

$$
\dot{N}=3 \times 10^{7} / \mathrm{s} .
$$

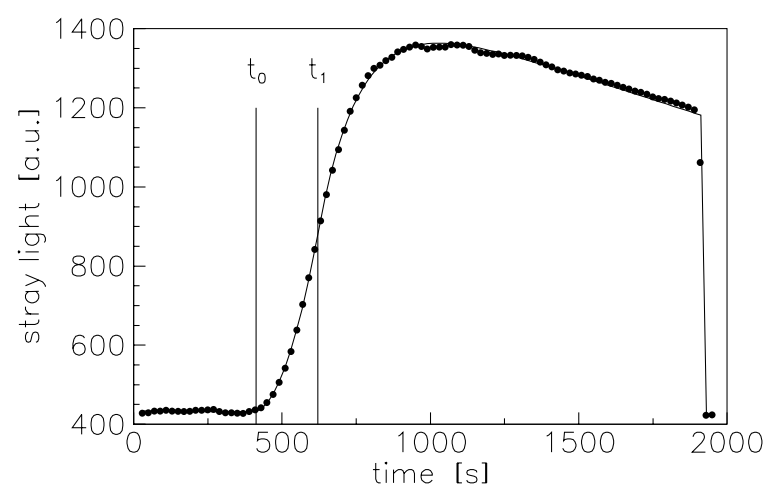

Fig. 7. Stray light curve for a $130 \AA \mathrm{D}_{2}$ film at a dewetting temperature of $4.4 \mathrm{~K}$ (circles) and fit according equation (24).

From Figure 4 we estimate for this case a time of growth of about $2000 \mathrm{~s}$ which yields with (18) a final number of $N=6 \times 10^{10}$ molecules per cluster on the average, corresponding to a cluster radius of

$$
a \approx 1 \mu \mathrm{m}
$$

in qualitative agreement with earlier observations $[20,21]$. Considering the total average of 47 monolayers $(\approx 160 \AA)$, one further calculates an average cluster density of

$$
\mathrm{d} Z / \mathrm{d} A \approx 8 \times 10^{9} / \mathrm{m}^{2} .
$$

\subsection{Stray light signal and its parametrisation}

We may also estimate the stray light intensity by help of this model. Starting from a calculation of Mie scattering by dielectric spheres in reference [23] one estimates a differential cross section of the final spheres at $60^{\circ}$ scattering angle of about $\mathrm{d} \sigma\left(\theta=60^{\circ}\right) / \mathrm{d} \Omega=\mathcal{O}\left(10^{-13} \mathrm{~m}^{2}\right)$. (Scattering by $120^{\circ}$ from the incoming beam (see Fig. 2) is much weaker in comparison.) Inserting the solid angle of detection $\Delta \Omega \approx 10^{-2}$ and the density of scattering centres (20) one arrives at a ratio of stray light intensity over the incoming one of

$$
I_{\text {stray }} / I_{0}=\mathcal{O}\left(10^{-5}\right) .
$$

This is the right order of magnitude which has been observed; although it could not be checked quantitatively with the given set up.

More detailed information about the cluster growth is obtained from a parametrisation of the stray light signal as function of time $t$ (see Fig. 7). It turns out that the first rise of stray light after warming up the film to the chosen dewetting temperature can always be fitted by a second order parabola. In this initial phase we may assume the cluster size to be still much smaller than the wavelength $(a \ll \lambda)$. This is the regime of Rayleigh scattering in which the cross section rises with the sixth power of $a$

$$
\sigma \propto a^{6}(2 \pi / \lambda)^{4}
$$


Since $\sigma$ is observed to grow as $t^{2}, a$ is got to grow as $t^{1 / 3}$ in order to fulfil (22). With $a$ being proportional to $N^{1 / 3}$ we conclude that the clusters grow linearly in time

$$
N \propto t
$$

in accordance with our schematic model.

At later times the growth of stray light slows down for two reasons mainly: (i) because the cluster size approaches the regime of Mie scattering where the cross section rises as $a^{2}$ only, (ii) because the quench-condensed film material is getting exhausted. We model this phase by an asymptotic growth term $\sim\left(1-\mathrm{e}^{-\gamma t}\right)$. At higher dewetting temperatures we have also to consider that desorption occurs already during dewetting. We take this effect into account by a term of linear signal decrease $\left.\sim\left(1-\dot{d} / d_{0}\right) t\right)$. Collecting all terms we arrive at the following model function of the stray light signal

$$
\begin{aligned}
S(t)= & \left(A\left(t-t_{0}\right)^{2} \theta\left(t-t_{0}\right) \theta\left(t_{1}-t\right)\right. \\
& \left.+\left(A\left(t_{1}-t_{0}\right)^{2}+B\left(1-\mathrm{e}^{-\gamma\left(t-t_{1}\right)}\right)\right) \theta\left(t-t_{1}\right)\right) \\
& \times\left(1-\frac{\dot{d}}{d_{0}} t\right) .
\end{aligned}
$$

The $\theta$-functions in (24) define the times $t_{0}$ and $t_{1}$ when the parabolic signal growth and the asymptotic one appears on the time scale, respectively ${ }^{4}$. The measurement started at $t=0$. The parameters $t_{0}, t_{1}, A, B, \gamma, \dot{d} / d_{0}$ are fitted to the signal. An example is shown in Figure 7 for the case of $\mathrm{D}_{2}$ dewetting at $4.4 \mathrm{~K}$. The fit turns out to be very good.

\subsection{Evaluation of activation energies}

We may take the fit value defined above of $t_{1}-t_{0}$ as the time interval $\Delta t$ as defined in (15). According to (16) it should scale with temperature as $\mathrm{e}^{\Delta E / k_{\mathrm{B}} T}$, which gives a handle to determine the activation energy in principle. As long as desorption is still negligible, also the total stray light curve should scale in time according to (16) for different temperatures. As an example Figure 8 shows the stray light curve of an $\mathrm{D}_{2}$-film dewetting at $T_{1}=3.8 \mathrm{~K}$ and being rescaled in time by a scaling factor $s=0.39$ to fit $\mathrm{D}_{2}$ stray light data obtained at the higher temperature of $T_{0}=4.2 \mathrm{~K}$. More generally, Figure 9 shows in an Arrhenius plot $\ln s_{\alpha i}$ versus $1 / T$ of the measured scaling factors $s_{\alpha i}$ for the different molecules $\alpha$ at temperature $T_{i}$ compared to $\mathrm{D}_{2}$ at temperature $T_{0}=4.2 \mathrm{~K}$. The slope of each series is the activation energy $\Delta E_{\alpha}$ of this species in units of $k_{\mathrm{B}} \mathrm{K}$. In view of the relatively large error bars and the scatter of the few measured points in each of the series, however, the lever arm of each single series is a bit short for yielding a precise result. More stable values can

\footnotetext{
${ }^{4}$ We neglect here the very late stage of the dewetting process, which is characterised by coarse graining or "Ostwald ripening" of the clusters. This process is to be expected to occur on time scales much longer than investigated here.
}

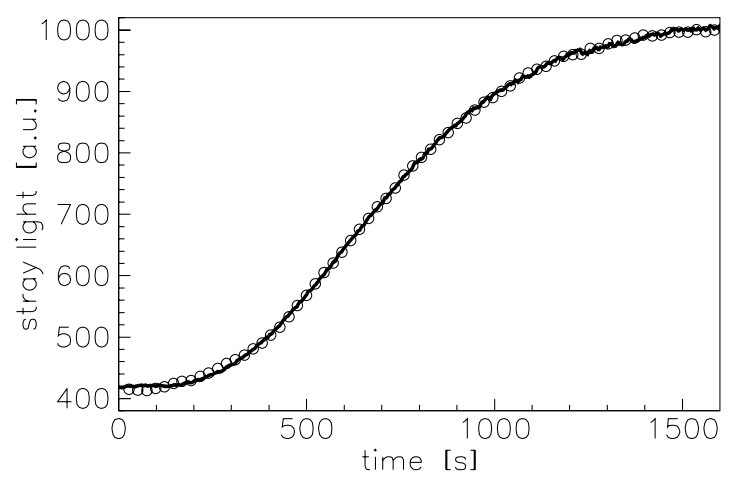

Fig. 8. Stray light curve of an $\mathrm{D}_{2}$-film dewetting at $T_{1}=3.8 \mathrm{~K}$ (circles) and being rescaled in time by a scaling factor $s=0.39$ to fit $\mathrm{D}_{2}$ stray light data obtained at the higher temperature of $T_{0}=4.2 \mathrm{~K}$ (line).

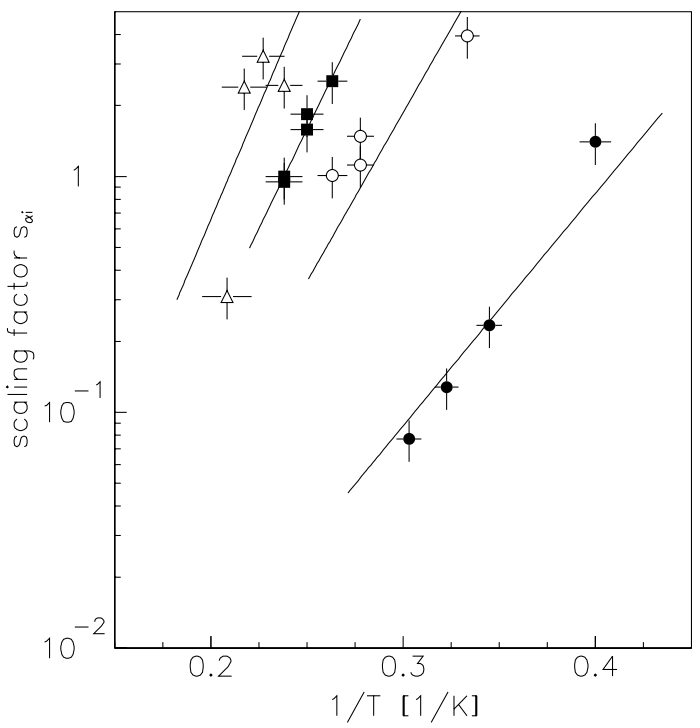

Fig. 9. Arrhenius plot with scaling factors $s_{\alpha i}$ of dewetting times versus $1 / T$ for the isotopes $\mathrm{H}_{2}$ (dots), $\mathrm{HD}$ (circles), $\mathrm{D}_{2}$ (filled squares), and $\mathrm{T}_{2}$ (triangles), normalised to $\mathrm{D}_{2}$ at $T_{0}=$ $4.2 \mathrm{~K}$. The solid lines show the common fit according to (27).

be obtained in a general fit of all scaling factors $s_{\alpha i}$ in Figure 9, including those between different species. According to our model the characteristic times of growth for a given film thickness differ only by their diffusion constants (see (15)). We can, therefore, introduce those ratios as additional constraints into the overall fit. We obtain from (15, $7,5)$ for the scaling factors

$$
\begin{aligned}
s_{\alpha i} & =\frac{\Delta t_{\alpha}\left(T_{i}\right)}{\Delta t_{\mathrm{D}_{2}}\left(T_{0}\right)} \\
& =\frac{D_{\mathrm{D}_{2}}\left(T_{0}\right) n_{\mathrm{sD}_{2}}(b)}{D_{\alpha}\left(T_{i}\right) n_{\mathrm{s} \alpha}(b)} \\
& =\sqrt{\frac{\Delta E_{\mathrm{D}_{2}} g_{\alpha}^{2} m_{\alpha}}{\Delta E_{\alpha} g_{\mathrm{D}_{2}}^{2} m_{\mathrm{D}_{2}}}} \exp \left(\frac{\Delta E_{\alpha}}{k_{\mathrm{B}} T_{i}}-\frac{\Delta E_{\mathrm{D}_{2}}}{k_{\mathrm{B}} T_{0}}\right) .
\end{aligned}
$$

It is seen from (27) that the scaling factors are determined primarily by the ratios of the respective Boltzmann 
Table 1. Activation energies for surface diffusion

\begin{tabular}{ccccc}
\hline \multicolumn{4}{c}{$\Delta E\left[k_{\mathrm{B}} \mathrm{K}\right]$} & method \\
\hline $\mathrm{H}_{2}$ & $\mathrm{HD}$ & $\mathrm{D}_{2}$ & $\mathrm{~T}_{2}$ & \\
\hline \hline $23.3_{-3.9}^{+3.6}{ }_{-1.8}^{+1.6}$ & $34.3_{-4.0}^{+3.7}{ }_{-2.2}^{+1.9}$ & $39.5_{-5.1}^{+4.8}{ }_{-2.2}^{+2.1}$ & $44.8_{-5.6}^{+5.2}{ }_{-2.8}^{+2.2}$ & stray light, this work \\
\hline $23 \pm 2$ & $35 \pm 3$ & $47 \pm 3$ & & Rayleigh waves [11] \\
\hline $23.9 \pm 0.3$ & \multicolumn{4}{c}{ plasmons [21,22] } \\
\hline
\end{tabular}

factors, i.e. in the plot of Figure 9 they are approximately given by

$$
\left.\ln \left(s_{\alpha i}\right) \approx f+\frac{\Delta E_{\alpha}}{k_{\mathrm{B}} T_{i}}, \quad \text { (with } f \approx \text { const. }\right) .
$$

The result of the common fit is shown by the straight lines in Figure 9. The resulting activation energies $\Delta E_{\alpha}$ are given in Table 1 together with results obtained from previous measurements using different methods. The agreement is satisfactory.

We apply to each scaling factor $s_{\alpha i}$ a relative uncertainty of $20 \%$, which is larger than their statistical uncertainty from the scaling fit, to account for small shape and film thickness variations between the measurements. For the temperature measurement we estimate its uncertainty by considering the quadratic sum of a minimal uncertainty of $0.05 \mathrm{~K}$ and a temperature uncertainty which comes from a constant uncertainty $\Delta R$ of the resistance measurement of our carbon glass sensor. Applying these uncertainties to our data, the common fit according to (27) yields a $\chi^{2} /$ d.o.f. $=1.1$. As systematic uncertainty we take into account a common temperature calibration uncertainty of $0.1 \mathrm{~K}$ and a variation of $\Delta R$ by $40 \%$.

Since the scaling factors $s_{\alpha i}$ correspond to the time of growth of a molecule $\alpha$ at temperature $T_{i}$ normalised to the time of growth of $\mathrm{D}_{2}$ clusters $\Delta t_{\mathrm{D}_{2}}(T=4.2 \mathrm{~K}) \approx$ $1200 \mathrm{~s}$ measured at $T_{0}=4.2 \mathrm{~K}$ (see Fig. 8) we may extrapolate from Figure 9 for our case of interest, a $T_{2^{-}}$ film, a dewetting time of

$$
\begin{array}{rlr}
\ln \left(\Delta t_{T_{2}}(T=1.86 \mathrm{~K}) / \mathrm{s}\right) & =21.3_{-1.8}^{+1.6}{ }_{-0.7}^{+0.1} \\
\Delta t_{T_{2}}(T=1.86 \mathrm{~K}) & >8.0 \mathrm{a} \quad(68 \% \text { C.L. }) \\
\Delta t_{T_{2}}(T=1.86 \mathrm{~K}) & >1.2 \mathrm{a} \quad(95 \% \text { C.L. })
\end{array}
$$

at the working temperature $T=1.86 \mathrm{~K}$ of our present, improved source. For an isotopically pure $\mathrm{T}_{2}$ source (or for one with a higher tritium purity, e.g. our present sources having $90 \%$ tritium content) this time is expected to be still longer. This extrapolation is valid if the influence of the radioactivity of tritium does not play a significant role in the dewetting process. There is no experimental indication of a roughening of tritium films at such low temperatures: A tritium film kept over 4 days at $1.7 \mathrm{~K}$ did not show any increase of scattered light. Moreover $\beta$ spectra of tritium films prepared and kept at $1.86 \mathrm{~K}$ over more than 4 weeks did not exhibit any change of shape with time. They could be described consistently by taking into account the energy loss for a homogeneously flat film, in contrast to the spectra of previous measurements with tritium films kept at about $3 \mathrm{~K}$ and briefly even at $4.2 \mathrm{~K}[5,24]$.

\subsection{Competition by tunnelling}

In view of the low activation energy $\Delta E$ of the hopping process, e.g. $43 \mathrm{~K}$ for $\mathrm{D}_{2}$, one may think of quantummechanical tunnelling as a competing process. A rough calculation [14] yields in fact for $\mathrm{D}_{2}$ a tunnelling time constant of only $10^{-8} \mathrm{~s}$, ten times shorter than obtained from Frenkel's law at our particular temperature (see (9)). One thus ought to observe temperature independent dewetting. This, however, is contradicted by experiment. A similar discrepancy is known for volume diffusion of $\mathrm{H}_{2}$ and $\mathrm{D}_{2}$ where the predicted tunnelling was not observed [25]. It seems that a naive tunnelling calculation with just the measured activation energy and the free particle mass as input parameters oversimplifies the dynamics in which the next neighbours are involved as well as the hopping or tunnelling molecule.

\section{Conclusion}

We have observed dewetting and clustering of quenchcondensed films of hydrogen molecules $\mathrm{H}_{2}, \mathrm{HD}, \mathrm{D}_{2}, \mathrm{~T}_{2}$ from different substrates and as a function of temperature. For none of the substrates tried out, aluminium, carbon or sandwiched ones with some other van der Waals film in between, (noble gases, $\mathrm{CH}_{4}$ ), the wetted phase was found to be energetically stable in thermal equilibrium, but dewetting was observed always in the temperature region just below desorption. We have reported this finding already in a foregoing paper [8]. Although our hopes to realize a thermodynamically stable $\mathrm{T}_{2}$-film for the purpose of precision measurements of the $\beta$-spectrum from such a source were not fulfilled, we learned from the present study that the dewetting dynamics is at least sufficiently slow at temperatures $T<2 \mathrm{~K}$ to allow long term measurements on quench-condensed $\mathrm{T}_{2}$-films without the risk of structural changes. This way our primary motivation for these studies could be satisfied.

We have studied two different signatures of the dewetting and clustering process. The first is directly connected to our $\beta$-decay experiments and concerns the measurement of energy loss of energetic electrons $(E \approx 18 \mathrm{keV})$ in such dewetted films. We have observed that the energy loss spectrum gets harder due to the enhanced chance 
of multiple scattering within the final clusters. The size of this effect was found to be just right to explain the measured deterioration of the $\mathrm{T}_{2} \beta$-spectrum and its impact on the determination of the neutrino mass.

The second method, stray light measurement from the film, allowed to observe the cluster growth in real time and, therefore, was favoured for the dynamical studies. Dewetting time constants have been measured in the range of a couple of minutes to several hours. The acceleration with temperature follows the Arrhenius law and yields activation energies for the different isotopes. Coverage of the quench-condensed hydrogen films by a few monolayers of more tightly bound van der Waals substances like Ar or $\mathrm{CH}_{4}$ suppressed dewetting completely and thus proved it to be a surface diffusion process $[8,14]$.

Based on these findings we have developed a schematic model of the dewetting dynamics which assumes diffusional transport from the energetically disfavoured disordered film to energetically favoured crystalline clusters over the very thin residual film (few monolayers) which is tightly bound to the substrate. The diffusion constant is controlled by Frenkel's law for the hopping rate from one lattice site to the next. With some geometrical simplifications the diffusion equation can be solved analytically. It reproduces satisfactorily all observed dynamical features of the cluster growth including their final size.

This work was supported by the Deutsche Forschungsgemeinschaft under contract Ot33/13. We appreciate helpful discussions with H. Barth, R. Conradt, M. Gundlach and J. Klier.

\section{References}

1. S. Dietrich, in Phase Transitions and Critical Phenomena, edited by C. Domb, J. Lebowitz (Academic Press, London, 1988), Vol. 12, p. 1.

2. See, e.g., J. Krim, J.G. Dash, J. Suzanne, Phys. Rev. Lett. 52, 640 (1984); A.D. Migone, A. Hofmann, J.G. Dash, O.E. Vilches, Phys. Rev. B 37, 5440 (1988); P. Leiderer, U. Albrecht, J. Low Temp. Phys. 89, 229 (1992).

3. S. Herminghaus, J. Vorberg, H. Gau, R. Conradt, D. Reinelt, H. Ulmer, P. Leiderer, M. Przyrembel, Ann. Phys. (Leipzig) 6, 425 (1997).

4. Ch. Weinheimer, B. Degen, A. Bleile, J. Bonn, L. Bornschein, O. Kazachenko, A. Kovalik, E.W. Otten, Phys. Lett. B 460, 219 (1999).

5. Ch. Weinheimer, M. Przyrembel, H. Backe, H. Barth, J. Bonn, B. Degen, Th. Edling, H. Fischer, L. Fleischmann,
J.U. Groo, R. Haid, A. Hermanni, G. Kube, P. Leiderer, Th. Loeken, A. Molz, R.B. Moore, A. Osipowicz, E.W. Otten, A. Picard, M. Schrader, M. Steininger, Phys. Lett. B 300, 210 (1993).

6. M. Przyrembel, PhD Thesis, Mainz University, 1995.

7. F.T. Gittes, M. Schick, Phys. Rev. B30, 209 (1984).

8. L. Fleischmann, J. Bonn, B. Degen, M. Przyrembel, E.W. Otten, Ch. Weinheimer, P. Leiderer, J. Low Temp. Phys. (in print).

9. P. Leiderer, U. Albrecht, J. Low Temp. Phys. 89, 229 (1992).

10. R. Conradt, U. Albrecht, S. Herminghaus, P. Leiderer, Physica B 194-196, 679 (1994).

11. J. Classen, K. Eschenröder, G. Weiss, Ann. Phys. (Leipzig) 4, 1 (1995); Phys. Rev. B 52, 11475 (1995).

12. H. Menges, H.v. Löhneysen, J. Low Temp. Phys. 84 (1991) 237.

13. K.H. Müller, Surf. Sci. 184, L375 (1987).

14. L. Fleischmann, PhD Thesis, Mainz University, 1998.

15. A. Picard, H. Backe, H. Barth, J. Bonn, B. Degen, Th. Edling, R. Haid, A. Hermanni, P. Leiderer, Th. Loeken, A. Molz, R.B. Moore, A. Osipowicz, E.W. Otten, M. Przyrembel, M. Schrader, M. Steininger, Ch. Weinheimer, Nucl. Instr. Meth. B 63 (1992) 345.

16. A. Picard, H. Backe, J. Bonn, B. Degen, R. Haid, A. Hermanni, P. Leiderer, A. Osipowicz, E.W. Otten, M. Przyrembel, M. Schrader, M. Steininger, Ch. Weinheimer, Z. Phys. A 342 (1992) 71.

17. V.N. Aseev, A.I. Belesev, A.I. Berlev, E.V. Geraskin, O.V. Kazachenko, Yu.E. Kuznetsov, V.M. Lobashev, R.P. Ostroumov, N.A. Titov, S.V. Zadorozhny, Yu.I. Zakharov, J. Bonn, B. Bornschein, L. Bornschein, E.W. Otten, M. Przyrembel, Ch. Weinheimer, A. Saenz, Eur. Phys. J. D 10, 39 (2000).

18. I.F. Silvera, Rev. Mod. Phys. 52 (1980) 393.

19. R.W.G. Wyckoff, Crystal Structures (J. Wiley \& Sons, New York, 1963).

20. J. Classen, Dissertation, Heidelberg University, 1994.

21. R.N.J. Conradt, Dissertation, Konstanz University, 1996.

22. U. Albrecht, R. Conradt, S. Herminghaus, P. Leiderer, Fizika Nizkikh Tem. 22, 1 (1996).

23. H.C. van de Hulst, Light scattering by small particles (John Wiley \& Sons Inc., New York, 1957).

24. H. Backe, H. Barth, A. Bleile, J. Bonn, B. Degen, L. Fleischmann, M. Gundlach, E.W. Otten, M. Przyrembel, Ch. Weinheimer, Proc. XVII Conference on Neutrino Physics and Astrophysics, Neutrino 96, Helsinki/Finnland, June 1996 (World Scientific/Singapure).

25. C. Ebner, C.C. Sung, Phys. Rev. A 5, 2625 (1972). 